\title{
Role of diffusion-weighted magnetic resonance imaging in the differentiation of benign and malignant pulmonary lesions
}

\author{
Eman Abdelaziz Alnaghy ${ }^{1 A, B, C, D, E, F}$, Mahmoud Ahmed El-Nahas ${ }^{2 A}$, Ahmed Galal Sadek ${ }^{2 D}$, \\ Noureldin Noaman Gwely ${ }^{3 B}$, Mohamed Magdy Elrakhawy ${ }^{2 \mathrm{~F}}$ \\ 'Diagnostic Radiology, Faculty of Medicine, Mansoura University, Egypt \\ 2Diagnostic Radiology and Medical Imaging, Faculty of Medicine, Mansoura University, Egypt \\ ${ }^{3}$ Cardiothoracic Surgery, Faculty of Medicine, Mansoura University, Egypt
}

\section{Abstract}

Purpose: To evaluate the role of magnetic resonance (MRI) diffusion-weighted imaging (DWI) and apparent diffusion coefficient (ADC) measurement of solid and cystic pulmonary masses in differentiating benign from malignant lesions.

Material and methods: The study included 41 patients with pulmonary masses, who underwent conventional MRI and DWI ( $b$ value 0,500 , and $1000 \mathrm{~s} / \mathrm{mm}^{2}$ ) examinations with 1.5 -T MRI. The diffusion signal and the mean ADC values of the solid and cystic lesions were obtained. Statistical analyses were performed with the Mann-Whitney $U$ test (z), Pearson's chi-square test, and receiver operating characteristic (ROC) analysis.

Results: Thirty-three lesions were malignant, and eight lesions were benign. The malignant masses showed significantly higher signal intensity on DWI than benign masses $(p=0.006)$, and the mean ADC value of malignant solid lesions was significantly lower than that of benign lesions $\left(p=0.02\right.$ ). By ROC analysis, an ADC cut-off value of $1.4 \times 10^{-3} \mathrm{~mm}^{2} / \mathrm{s}$ was considered the threshold value, and the sensitivity and specificity were $93.8 \%$ and $75 \%$, respectively. There was no significant difference between the ADC value of the cystic parts inside the benign and the malignant lesions.

Conclusions: Diffusion-weighted MRI and measurement of ADC value can significantly differentiate between solid benign and malignant pulmonary masses.

Key words: diffusion MRI, ADC, benign, malignant, pulmonary lesions.

\section{Introduction}

Diffusion magnetic resonance imaging (MRI) detects the random motion of water molecules in biological tissues (Brownian motion) and allows characterisation of tissue microstructural changes. Water diffusion is changed in various disease processes reflecting physiological and morphological tissue criteria such as cell density and tissue viability [1].

This can be quantified by apparent diffusion coefficient $(\mathrm{ADC})$ value. Calculating the ADC determines different degrees of biological aggressiveness; ADC values of benign lesions are significantly higher than those of malignant lesions [2].
Morphologic analysis is not sufficient for differentiating malignant from benign pulmonary nodules. When the nodules have significant enhancement, overlap occurs between active granulomas, hypervascular benign lesions, and malignant lesions [3].

Computed tomography (CT) and positron emission tomography (PET) are common noninvasive methods used to evaluate pulmonary nodules. PET can differentiate malignant from benign lesions. But it has given false-negative results for well-differentiated pulmonary adenocarcinoma and false-positive results for inflammatory nodules [3].

Also, PET-CT is associated with a considerable radiation burden to patients and medical personnel [4].

Correspondence address:

Dr. Eman Abdelaziz Alnaghy, Diagnostic Radiology, Faculty of Medicine, Mansoura University, Egypt, e-mail: emnaghi@hotmail.com

Authors' contribution:

A Study design · B Data collection · C Statistical analysis · D Data interpretation · E Manuscript preparation · F Literature search · G Funds collection 
The advantage of diffusion-weighted imaging (DWI) is that it is completely noninvasive, does not require exposure to ionising radiation, and does not cause patient discomfort [1].

\section{Aim of the study}

The aim of this work is to evaluate the role of MR diffusion imaging and ADC measurement in differentiating benign from malignant lung masses.

\section{Material and methods}

\section{Materials}

The study was done at the MRI unit of the Radiology Department of Mansoura University Hospital (MUH) between November 2015 and March 2018 and included 41 patients with pulmonary masses. Patients underwent MRI study followed by pathological assessment.

The entry criteria for patients included CT evidence of pulmonary mass or nodule greater than $2 \mathrm{~cm}$ in diameter, with exclusion of small sized lesions due to difficult interpretation of results.

\section{MRI technique}

All MR examinations were performed using 1.5-T MRI with a body phased-array coil, and patients were in a supine position. Sequences included conventional T1 and T2-WI, T2 fat sat images, and DWI.

T1-weighted fast spin echo sequence was obtained with the following parameters: TR/TE, $475.3 \mathrm{~ms} / 15 \mathrm{~ms}$; number of signals acquired, 1; field of view, $36 \mathrm{~cm}$; slice thickness, $6 \mathrm{~mm}$; gap, $1 \mathrm{~mm}$; matrix, $269 \times 222$; flip angle, $70^{\circ}$.

Respiratory gated T2-weighted fast spin echo sequence was obtained using the following parameters: TR/TE, $1250 \mathrm{~ms} / 80 \mathrm{~ms}$; echo train length, 80 ; number of signals acquired, 1; matrix, $328 \times 281$; field of view, $36 \mathrm{~cm}$; slice thickness, $6 \mathrm{~mm}$; gap, $1 \mathrm{~mm}$.

Diffusion gradients were applied in the three orthogonal directions, using a single-shot echo planar imaging sequence. DWIs were acquired with $b$ values of 0,500 , and $1000 \mathrm{~s} / \mathrm{mm}^{2}$. It had the following parameters: TR/TE, $2027 \mathrm{~ms} / 70 \mathrm{~ms}$; number of averages, 2; matrix, $168 \times 168$; field of view, $36 \mathrm{~cm}$; slice thickness, $6 \mathrm{~mm}$; gap, $6.6 \mathrm{~mm}$; flip angle, $90^{\circ}$; echo train length, 77 .

ADC maps were automatically generated by the software on the basis of the images obtained. ADC was calculated by drawing elliptical regions of interest (ROI) with an average size of $25 \pm 5$ voxels.

\section{Image analysis}

Diffusion images were analysed by qualitative and quantitative methods.
1. Qualitative analysis: On DWI the lesions were classified into lesions with low signal and lesions with high signal, compared to the signal intensity of adjacent skeletal muscles. The lesion signal on DWI was correlated with the signal on the ADC maps.

2. Quantitative analysis: ADC values were calculated from the ADC map. For calculating the mean ADC value of the solid part of the lesion, an ROI was placed on the solid area, with exclusion of any necrotic parts.

And for calculating the mean ADC value of the cystic part, an ROI was placed on the cystic area. For each procedure the ADC measurement was repeated three times, and the mean value was obtained.

\section{Statistical analysis}

Statistical analysis was performed using SPSS version 16. Parametric data were expressed in mean \pm standard deviation; non-parametric data were expressed in median and range. Pearson's chi-square test was used to compare the signal intensity of malignant and benign lesions on DWI.

To compare the mean ADC value of benign and malignant lesions, the independent samples $t$-test was used. Also, the Mann-Whitney $U$ test $(\mathrm{z}$ ) was used to compare non-parametric continuous variables in two different groups. Receiver operating characteristic (ROC) analysis was used to evaluate the diagnostic capability of ADC for differentiation between malignant and benign lesions. A value equal to or higher than the threshold was considered to indicate a benign lesion, and a value below the threshold was considered to indicate a malignant lesion. $P$ values less than 0.05 were considered to be statically significant.

\section{Results}

\section{Demographic data}

Our study included 41 patients with pulmonary masses: eight were benign and 33 were malignant. The benign lesions included three males and five females, with mean age $47 \pm 18.4$ years, age range 17-70 years. The malignant lesions included 22 males and 11 females, with mean age $51.3 \pm 13.7$ years, age range $19-70$ years.

The main complaints of the patients included dyspnoea, cough, fever, haemoptysis, and chest pain. One case was presented by bronchorrhoea.

\section{Signal intensity of the lesions}

Lesions were classified into lesions with low T1 signal and high T2 signal $(n=36)$, lesions with intermediate signal on T2 $(n=3)$, and lesions with high signal on T1 and T2-WI $(n=2)$. Fat sat images confirmed the absence of fat content in the lesions because all of them were hyperintense. 


\section{The nature of the pulmonary masses included in the study}

Lesions were classified into: solid, cystic, and solid with cystic parts. Most of the malignant lesions were solid, while half of the benign lesions were cystic (Table 1).

\section{Pathological diagnoses and associated findings of pulmonary masses included in this study}

The final diagnosis of the lesions was confirmed by histopathological examination. The lesions were divided into two groups: benign lesions $(n=8)$ and malignant lesions $(n=33)$, which included primary pulmonary carcinomas $(n=17)$ and metastatic tumours $(n=16)$. Detailed pathological diagnoses are listed in Table 2.

\section{Associated findings}

Five cases were associated with enlarged mediastinal lymph nodes (LNs) with restricted diffusion pattern. Four cases proved to be central bronchogenic carcinoma
Table 1. Nature of studied group of pulmonary cases (41 cases)

\begin{tabular}{|l|c|c|}
\hline Nature of the lesion & Benign $(n=8)$ & Malignant $(n=33)$ \\
\hline Solid & $3(37.5 \%)$ & $26(78.7 \%)$ \\
\hline Cystic & $4(50 \%)$ & $1(3 \%)$ \\
\hline Solid with cystic parts & $1(12.5 \%)$ & $6(18 \%)$ \\
\hline
\end{tabular}

and associated with distal lung collapse. Three cases were associated with bone metastasis including rib and chest wall metastasis. Three cases were associated with pleural effusion with free diffusion pattern.

\section{Signal intensity on DWI of the pulmonary masses included in this study}

Lesions were classified into lesions with low signal $(n=3)$ and lesions with high signal $(n=38)$, in comparison with the signal intensity of adjacent skeletal muscles (Table 3 ).

The malignant lesions showed high signal on DWI with restricted diffusion, while benign lesions showed variable appearance including low signal on DWI with free

Table 2. Pathological diagnoses and mean ADC values of pulmonary masses included in this study. Values with asterisk $\left({ }^{*}\right)$ are single values

\begin{tabular}{|c|c|c|c|}
\hline Diagnosis of pulmonary mass & $\begin{array}{l}\text { Frequency } \\
(n=41)\end{array}$ & $\begin{array}{l}\text { Mean ADC value } \pm \text { SD of solid lesions } \\
\qquad\left(\times 10^{-3} \mathrm{~mm}^{2} / \mathrm{s}\right)\end{array}$ & $\begin{array}{l}\text { Mean ADC value } \pm \text { SD of cystic lesions } \\
\qquad\left(\times 10^{-3} \mathrm{~mm}^{2} / \mathrm{s}\right)\end{array}$ \\
\hline Benign & 8 & $1.72 \pm 0.72$ & $1.46 \pm 0.51$ \\
\hline Inflammatory lesions & 2 & $2.2^{*}$ & $0.9^{*}$ \\
\hline Low-grade neuroendocrine tumour (carcinoid) & 2 & $1.9 \pm 0.63$ & NA \\
\hline Pyogenic abscess & 3 & $0.8^{*}$ & $1.4 \pm 0.41$ \\
\hline Sequestration cyst & 1 & NA & $2.1^{*}$ \\
\hline Malignant & 32 & $1.097 \pm 0.19$ & $2.38 \pm 0.3$ \\
\hline A. Primary lung cancer & 17 & $1.10 \pm 0.18$ & $2.4 \pm 0.36$ \\
\hline Adenocarcinoma & 13 & $1.04 \pm 0.18$ & 2.7 \\
\hline Bronchioalveolar carcinoma & 1 & $1.3^{*}$ & NA \\
\hline Small cell lung cancer & 2 & $1.25 \pm 0.07$ & $2.25 \pm 0.35$ \\
\hline Squamous cell lung cancer & 1 & $1.3^{*}$ & NA \\
\hline B. Metastatic masses & 16 & $1.093 \pm 0.19$ & $2.36 \pm 0.32$ \\
\hline Malignant nerve sheath tumour & 1 & $1.3^{*}$ & NA \\
\hline Malignant melanoma & 1 & $1.4^{*}$ & NA \\
\hline Adenocarcinoma & 3 & $0.83 \pm 0.23$ & NA \\
\hline Medullary thyroid cancer & 1 & $1^{*}$ & NA \\
\hline Prostatic carcinoma & 1 & $1.2^{*}$ & NA \\
\hline Testicular carcinoma & 1 & $1^{*}$ & NA \\
\hline Germ cell tumour & 1 & $1.2^{*}$ & NA \\
\hline Breast cancer & 3 & $1.1 \pm 0.11$ & $2.6^{*}$ \\
\hline Myxoid chondrosarcoma & 1 & $1.2^{*}$ & NA \\
\hline Lymphoma & 2 & $1.1 \pm 0.14$ & $2.5^{*}$ \\
\hline Adenoid cystic carcinoma & 1 & NA & $2^{*}$ \\
\hline
\end{tabular}


Table 3. Diffusion signal (b1000) of the pulmonary masses included in this study

\begin{tabular}{|l|c|c|}
\hline Diffusion signal & Frequency $(n=40)$ & Percentage \\
\hline Low signal & 3 & 7.3 \\
\hline High signal & 38 & 92.7 \\
\hline
\end{tabular}

Table 4. Comparison of signal intensity on DWI (b1000) between different pulmonary masses

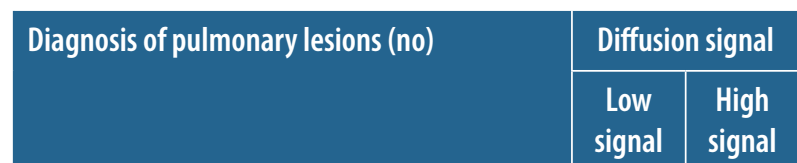

\begin{tabular}{|l|c|c|}
\hline \multicolumn{3}{|l|}{ Benign } \\
\hline Inflammatory (2) & 1 & 1 \\
\hline Low grade neuroendocrine tumour (carcinoid) (2) & 1 & 1 \\
\hline Pyogenic abscess (3) & 1 & 2 \\
\hline Sequestration cyst (1) & - & 1 \\
\hline Malignant & - & 7 \\
\hline Peripheral lung cancers (7) & - & 10 \\
\hline Central lung cancers (10) & - & 16 \\
\hline Metastatic lung cancer (16)
\end{tabular}

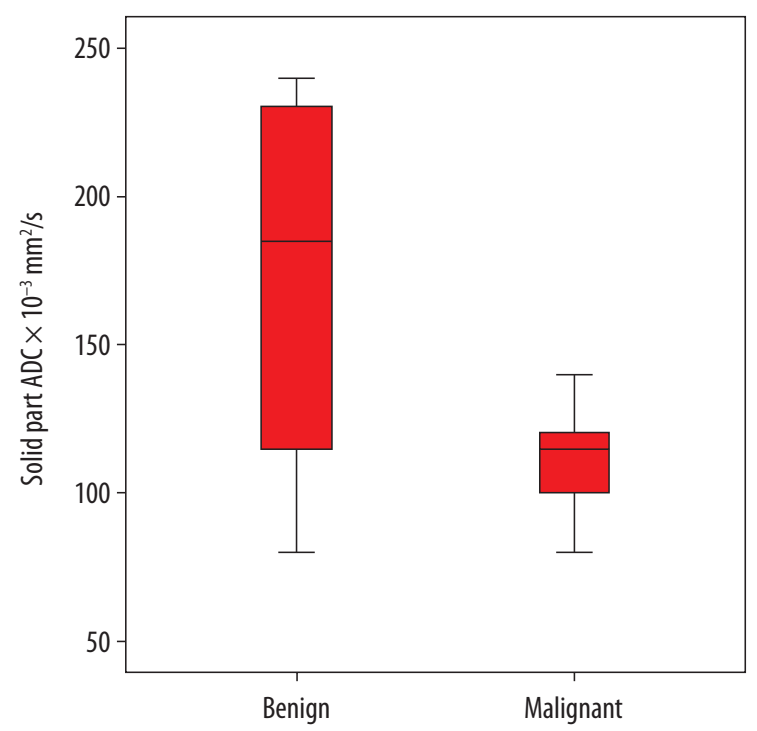

Figure 2. Comparison between mean ADC values of the solid benign and malignant pulmonary masses

diffusion and high signal on DWI (including true restriction and T2 shine-through artefact) (Figure 1).

Thirty-four cases had restricted diffusion including 31 malignant lesions and three benign lesions. Benign le-

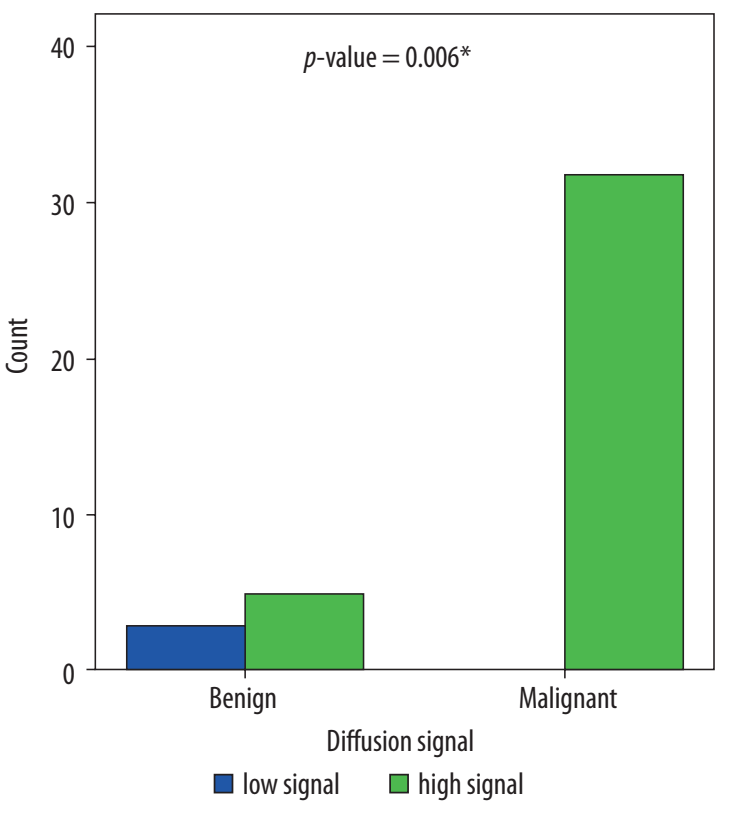

Figure 1. Comparison of DWI signal in malignant and benign pulmonary masses

sions with restricted diffusion were proved to be abscesses in two cases and a chronic inflammatory lesion with haemorrhage in one case.

Three cases had free diffusion; all were benign and were proved to be low-grade neuroendocrine tumour, chronic pyogenic abscess, and inflammatory granulomatous lesion.

Four cases had non-restricted diffusion (T2 shinethrough artefact), including two benign lesions (lowgrade neuroendocrine tumour and sequestration cyst) and two malignant lesions (metastatic adenoid cystic carcinoma of the submandibular gland and small cell lung cancer). A summarised comparison of signal intensity on DWI between different pulmonary masses is shown in Table 4.

\section{The mean $A D C$ value of the solid benign and malignant pulmonary masses}

The mean ADC value of benign lesions was $1.7 \pm 0.72$ $\times 10^{-3} \mathrm{~mm}^{2} / \mathrm{s}$, and for malignant lesions it was $1.09 \pm 0.0 .19$ $\times 10^{-3} \mathrm{~mm}^{2} / \mathrm{s}$, which was significantly lower than the benign lesions with $p$ value equal to 0.01 (Table 5 and Figure 2).

The area under the ROC curve was 0.84 (Figure 3 ). A cut-off value of $1.4 \times 10^{-3} \mathrm{~mm}^{2} / \mathrm{s}$ was considered to be the threshold. When an ADC value of $1.4 \times 10^{-3} \mathrm{~mm}^{2} / \mathrm{s}$ or more was estimated the lesion was considered to be benign, and a value below the threshold was considered

Table 5. Comparison between mean ADC value of the solid benign and malignant pulmonary masses

\begin{tabular}{|l|c|c|c|c|c|} 
& $n$ & ADC value $($ Mean \pm SD) & ADC value median (range) & $z$ & $p$-value \\
\cline { 1 - 3 } Benign & 4 & $1.7 \pm 0.72 \times 10^{-3} \mathrm{~mm}^{2} / \mathrm{s}$ & $1.8(1.6)$ & \multirow{2}{*}{$0.01^{*}$} \\
\cline { 1 - 3 } Malignant & 32 & $1.09 \pm 0.19 \times 10^{-3} \mathrm{~mm}^{2} / \mathrm{s}$ & $1.15(1)$ & \\
\hline
\end{tabular}


to indicate malignancy; the sensitivity and specificity were $93.8 \%$ and $75 \%$, respectively, and the $p$ value was equal to 0.02 (Table 6).

\section{The mean ADC value of cystic benign and malignant pulmonary masses}

Measuring the ADC value inside the entirely cystic lesions and inside the cystic or necrotic part of the mixed lesions containing solid and cystic components for both benign and malignant masses showed that the mean ADC value of benign lesions was $1.4 \pm 0.51 \times 10^{-3} \mathrm{~mm}^{2} / \mathrm{s}$ and for malignant lesions it was $2.38 \pm 0.3 \times 10^{-3} \mathrm{~mm}^{2} / \mathrm{s}$, which was statistically different from the benign masses (Table 7).

\section{Discussion}

Our study included 41 patients with pulmonary masses, including eight benign lesions and 33 malignant lesions.

A. Visual assessment: In our study the malignant lesions showed high signal on DWI with restricted diffusion pattern, and some benign lesions showed low signal on DWI with free diffusion pattern in agreement with the results obtained by Liu et al. [1], Satoh et al. [2], and Qi et al. [5].

However, we had three benign lesions with restricted diffusion pattern; they proved to be abscesses (two cases) and chronic inflammatory lesion with secondary haemorrhage (one case). Hence, we cannot depend on visual assessment only to judge the nature of a lesion, whether benign or malignant.

The restricted pattern in cases with pyogenic abscess was related to the highly viscous contents inside the abscess cavity, which limits the random molecular motion, resulting in high diffusion signal [6].

Whanga et al. [7] reported that late subacute haemorrhage shows an increase in DW signal and low ADC value.

Four cases had non-restricted diffusion pattern; the high signal in DWI was explained by the T2 shinethrough effect, which interferes with the viewing of diffusion images in isolation.

B. ADC values: Our study demonstrated that the mean $\mathrm{ADC}$ value of benign solid lesions was $1.7 \pm 0.72$ $\times 10^{-3} \mathrm{~mm}^{2} / \mathrm{s}$ and for malignant solid lesions it was 1.09 $\pm 0.19 \times 10^{-3} \mathrm{~mm}^{2} / \mathrm{s}$, which was significantly lower than that of the benign lesions. This result was in agreement with the results of Liu et al. [1] and Wu et al. [3].

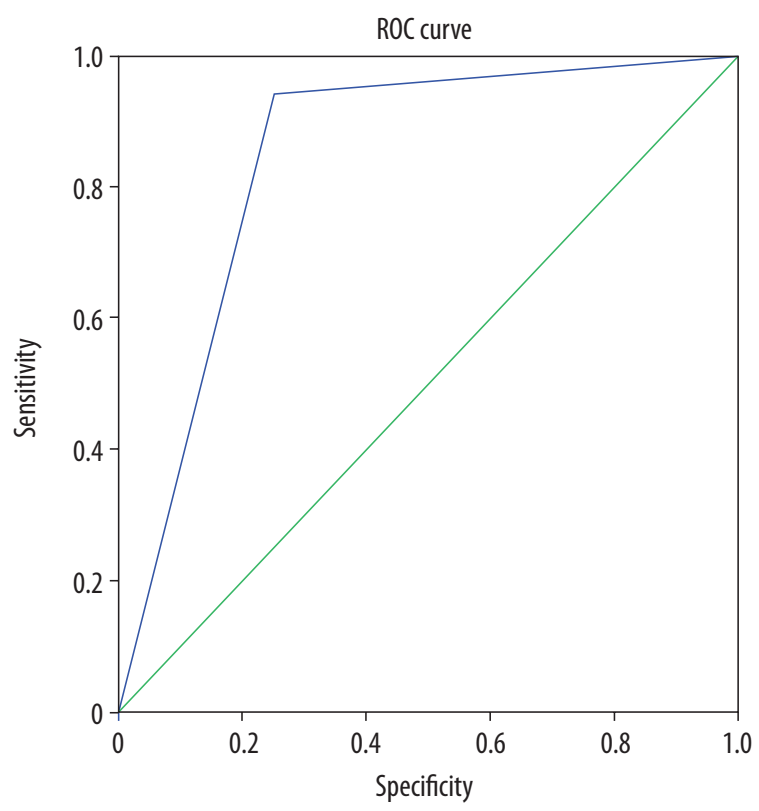

Figure 3. Receiver operating characteristic (ROC) curve of ADC value in solid benign and malignant pulmonary masses

Table 6. Receiver operating characteristic (ROC) of ADC value in solid benign and malignant pulmonary masses

\begin{tabular}{|l|c|}
\hline Area under the curve (AUC) & Solid part ADC \\
\hline Cutoff value & 0.84 \\
\hline$p$-value & 1.4 \\
\hline Sensitivity & $0.02^{*}$ \\
\hline Specificity & $93.8 \%$ \\
\hline Positive predictive value & $75 \%$ \\
\hline Negative predictive value & $25 \%$ \\
\hline
\end{tabular}

The area under the ROC curve was 0.84 , and a cut-off value $1.4 \times 10^{-3} \mathrm{~mm}^{2} / \mathrm{s}$ was considered to be the threshold; the sensitivity and specificity were $93.8 \%$ and $75 \%$, respectively. Our results were in agreement with Liu et al. [1], who reported the same cut-off value and considered a value of $1.400 \times 10^{-3} \mathrm{~mm}^{2} / \mathrm{s}$ to be the threshold; the sensitivity and specificity were $83.3 \%$ and $74.1 \%$, respectively.

Our results were conflicted with the results of Uto et al. [8], who reported that there was no significant difference between lung cancer and benign lesions based on the ADC value.

Table 7. Comparison between mean ADC value of cystic benign and malignant pulmonary masses

\begin{tabular}{|l|c|c|c|c|c|} 
& $n$ & ADC value (Mean \pm SD) & ADC value median (range) & z & -value \\
\cline { 1 - 4 } Benign & 5 & $1.4 \pm 0.51 \times 10^{-3} \mathrm{~mm}^{2} / \mathrm{s}$ & $1.3(1.2)$ & \multirow{2}{*}{$0.016^{*}$} \\
\cline { 1 - 3 } Malignant & 5 & $2.38 \pm 0.3 \times 10^{-3} \mathrm{~mm}^{2} / \mathrm{s}$ & $2.5(0.7)$ & \\
\hline
\end{tabular}



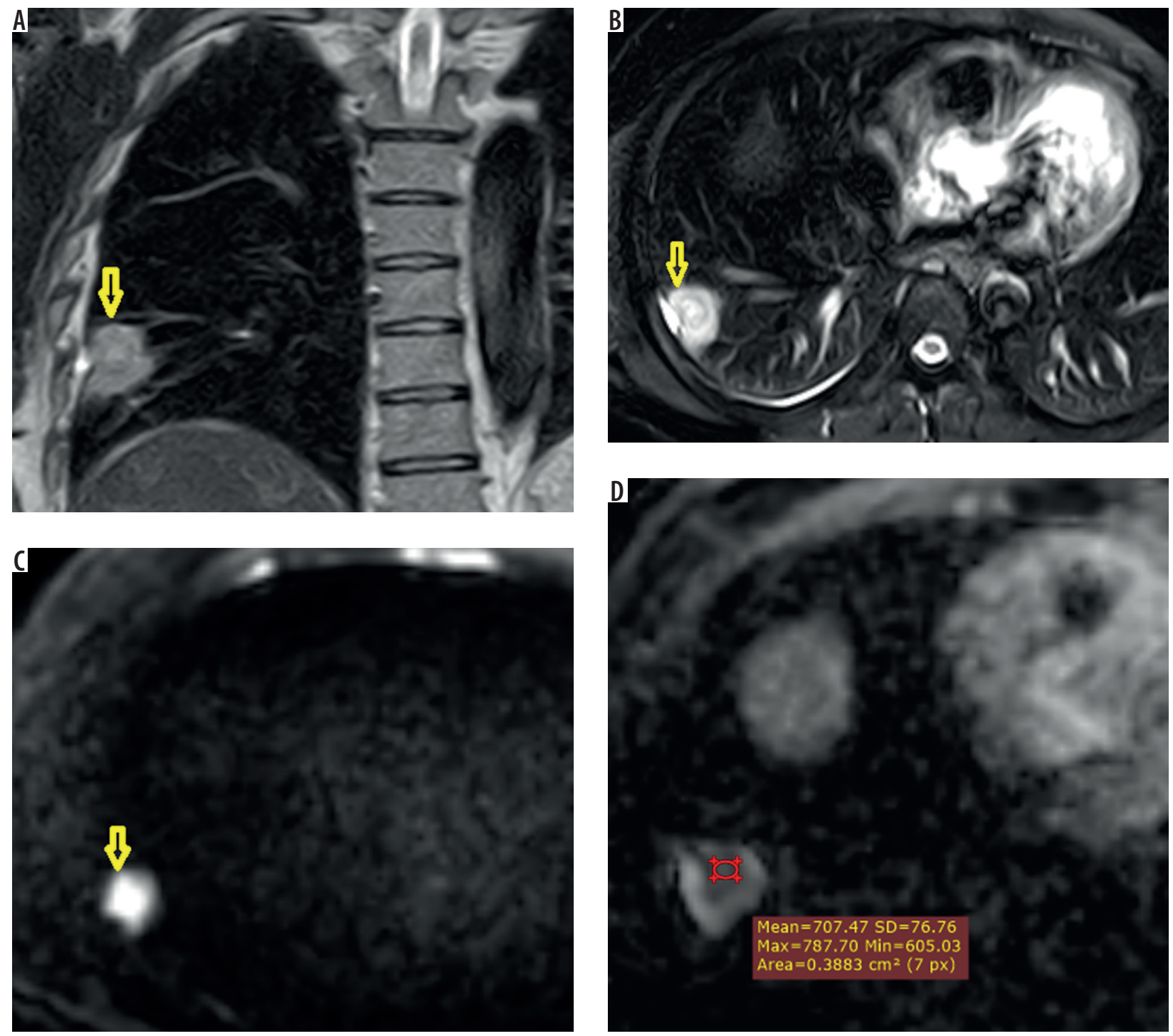

Figure 4. 55-year-old male patient presented by haemoptysis. A) Coronal T2-WI showing well defined mass at the RT lower lung lobe with hypointense signal. B) Axial T2-WI with fat saturation showing hyperintense signal of the mass with hyperintense signal at the adjacent part of the pleura. C) Axial DWI (b1000) showing strong hyperintense signal of the lesion. D) ADC map showing restricted diffusion of the mass with mean $A D C$ value $0.7 \times 10^{-3} \mathrm{~mm}^{2} / \mathrm{s}$. Pathological diagnosis was papillary adenocarcinoma

We had 17 cases of primary lung cancer, 13 of which were proven to be adenocarcinoma, one was proven to be bronchoalveolar carcinoma, two were proven to be small cell lung cancer, and one was proven to be squamous cell lung cancer.

The mean ADC value of adenocarcinoma cases was $1.04 \pm 0.18 \times 10^{-3} \mathrm{~mm}^{2} / \mathrm{s}$, which was lower than the mean ADC value of small cell lung cancer cases, which was $1.25 \pm 0.07 \times 10^{-3} \mathrm{~mm}^{2} / \mathrm{s}$ (Figure 4). This result was not in agreement with Liu et al. [1], who reported that the mean ADC value of small cell lung cancer was $1.06 \times 10^{-3}$ $\mathrm{mm}^{2} / \mathrm{s}$, which was lower than the mean ADC value of non-small cell lung cancer.

Among the 17 cases of primary lung cancer, we had four cases presented by hilar mass and distal collapse. In agreement with Sabri et al. [9], we found a significant difference between the malignant masses and the distal collapse as regard the signal on DWI and the mean ADC value. The hilar masses showed high signal on DWI with restricted diffusion pattern and mean $\mathrm{ADC}$ value 1.25 $\pm 0.36 \times 10^{-3} \mathrm{~mm}^{2} / \mathrm{s}$, while the distal collapse showed low signal on DWI with free diffusion pattern and mean ADC value $1.92 \pm 0.42 \times 10^{-3} \mathrm{~mm}^{2} / \mathrm{s}$ (Figure 5 )

We had 16 cases of metastatic pulmonary masses with mean ADC value $1.093 \pm 0.19 \times 10^{-3} \mathrm{~mm}^{2} / \mathrm{s}$, which was not significantly different from the mean ADC value of the primary pulmonary cancer that had a mean ADC value of $1.10 \pm 0.18 \times 10^{-3} \mathrm{~mm}^{2} / \mathrm{s}$.

We had one case of metastatic adenoid cystic carcinoma of the submandibular gland that showed multiple metastatic pulmonary deposits of cystic nature, with a mean ADC value of $2 \times 10^{-3} \mathrm{~mm}^{2} / \mathrm{s}$ (Figure 6).

This was in agreement with Karaman et al. [10], who reported that the mean ADC values of four adenoid 

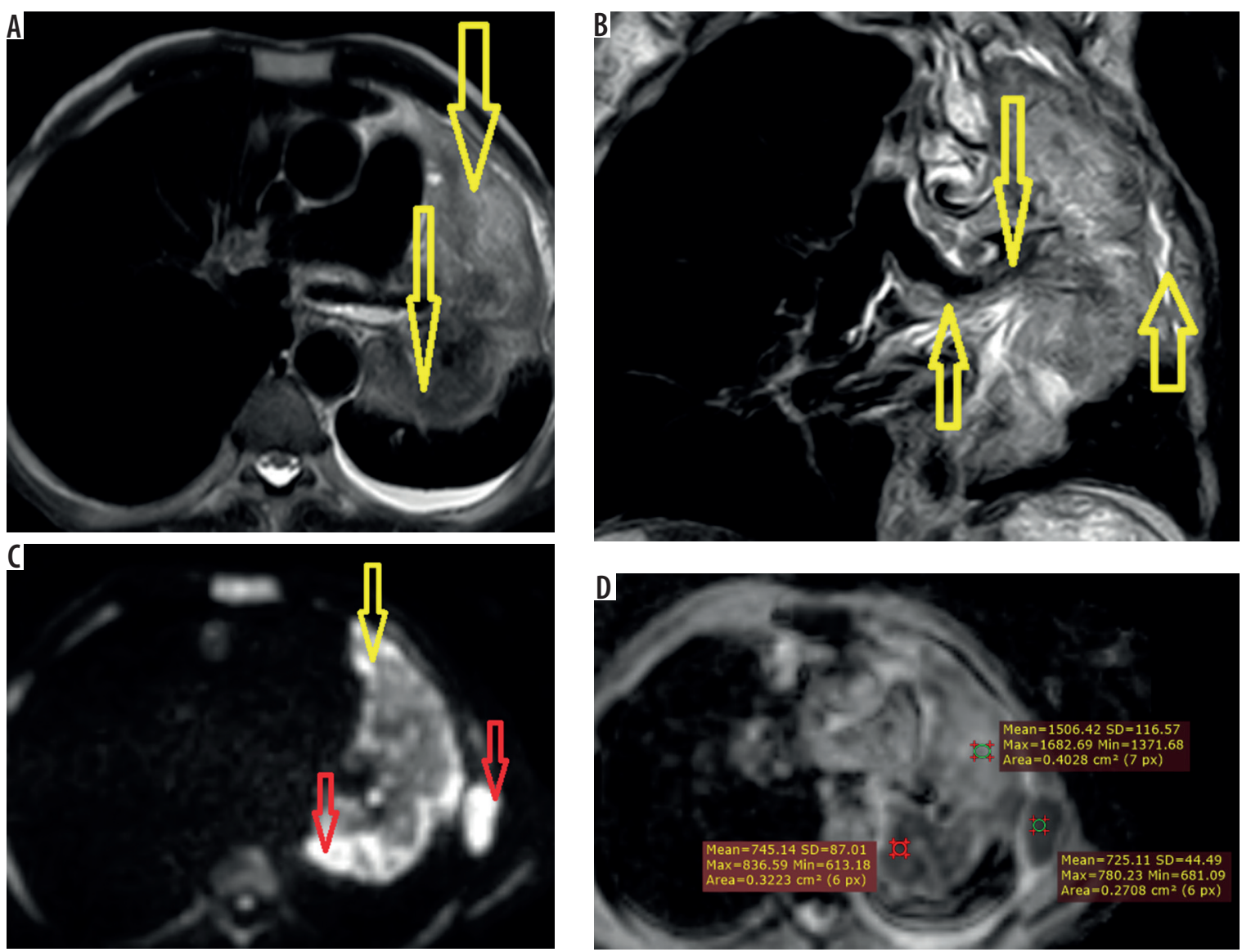

Figure 5. 65-year-old male with central bronchogenic carcinoma (adenocarcinoma). A) Axial T2-WI showing well-defined hyperintense mass at the hilum of the LT lung associated with collapsed LT upper lung lobe and mild LT sided pleural effusion. B) Coronal T2-WI the mass causes attenuation of the LT main pulmonary artery; the mass and the collapse are not separated from each other, with infiltration of the adjacent chest wall. C) Axial DWI (b1000) showing hypointense signal of the collapsed lung (yellow arrow) and hyperintense signal of the hilar mass and the infiltrated chest wall (red arrows). D) ADC map showing free diffusion of the collapse with mean $A D C$ value $1.5 \times 10^{-3} \mathrm{~mm}^{2} / \mathrm{s}$, while the mass and the infiltrated chest wall show restricted pattern with mean $A D C$ value $0.7 \times 10^{-3} \mathrm{~mm}^{2} / \mathrm{s}$

cystic carcinoma cases were higher than those of all malignant lesions, and the mean ADC value of adenoid cystic carcinoma overlapped with that of pleomorphic adenoma.

The mean ADC value of the low-grade neuroendocrine tumours included was $1.9 \pm 0.63 \times 10^{-3} \mathrm{~mm}^{2} / \mathrm{s}$, which was significantly higher than the mean ADC value of the primary as well as the metastatic pulmonary masses (Figure 7).

In our study we had five cases associated with mediastinal LNs; all of them had restricted diffusion pattern and low ADC value. The first case was presented by lung abscess and mediastinal lymph node enlargement, a biopsy from the mediastinal LNs revealed Hodgkin's lymphoma (Figure 8). Three cases were shown to be bronchogenic carcinoma with hilar and mediastinal LN enlargement. The last case was proven to be NHL. There was no significant difference between the mean ADC values of the enlarged mediastinal LNs in these cases. This was in agreement with the results of Abdel Razek et al. [11] and Gumustas et al. [12].
Associated pleural effusion was found in three cases. The mean $\mathrm{ADC}$ value of the pleural effusion was 3.1 $\pm 1.04 \times 10^{-3} \mathrm{~mm}^{2} / \mathrm{s}$.

Liver metastasis was seen in three cases; one of them proved to be bronchogenic carcinoma, the other two cases were metastatic adenocarcinoma and metastatic medullary thyroid carcinoma, the latter was presented by miliary pulmonary and hepatic metastases (Figure 9). The mean $\mathrm{ADC}$ value of the metastatic liver deposits was $0.86 \pm 0.2$ $\times 10^{-3} \mathrm{~mm}^{2} / \mathrm{s}$.

Bone and chest wall metastatic deposits were detected in three cases; two of them were shown to be bronchogenic carcinoma, and one was proven to be metastatic adenocarcinoma - the mean ADC value of the bony metastatic deposits was $0.83 \pm 0.32 \times 10^{-3} \mathrm{~mm}^{2} / \mathrm{s}$.

One case, metastatic breast cancer, was associated with malignant breast mass with a mean ADC value of $1 \times 10^{-3} \mathrm{~mm}^{2} / \mathrm{s}$.

Measurement of the ADC value inside the cystic or necrotic part of both benign and malignant lesions showed that the mean ADC value of benign lesions was 

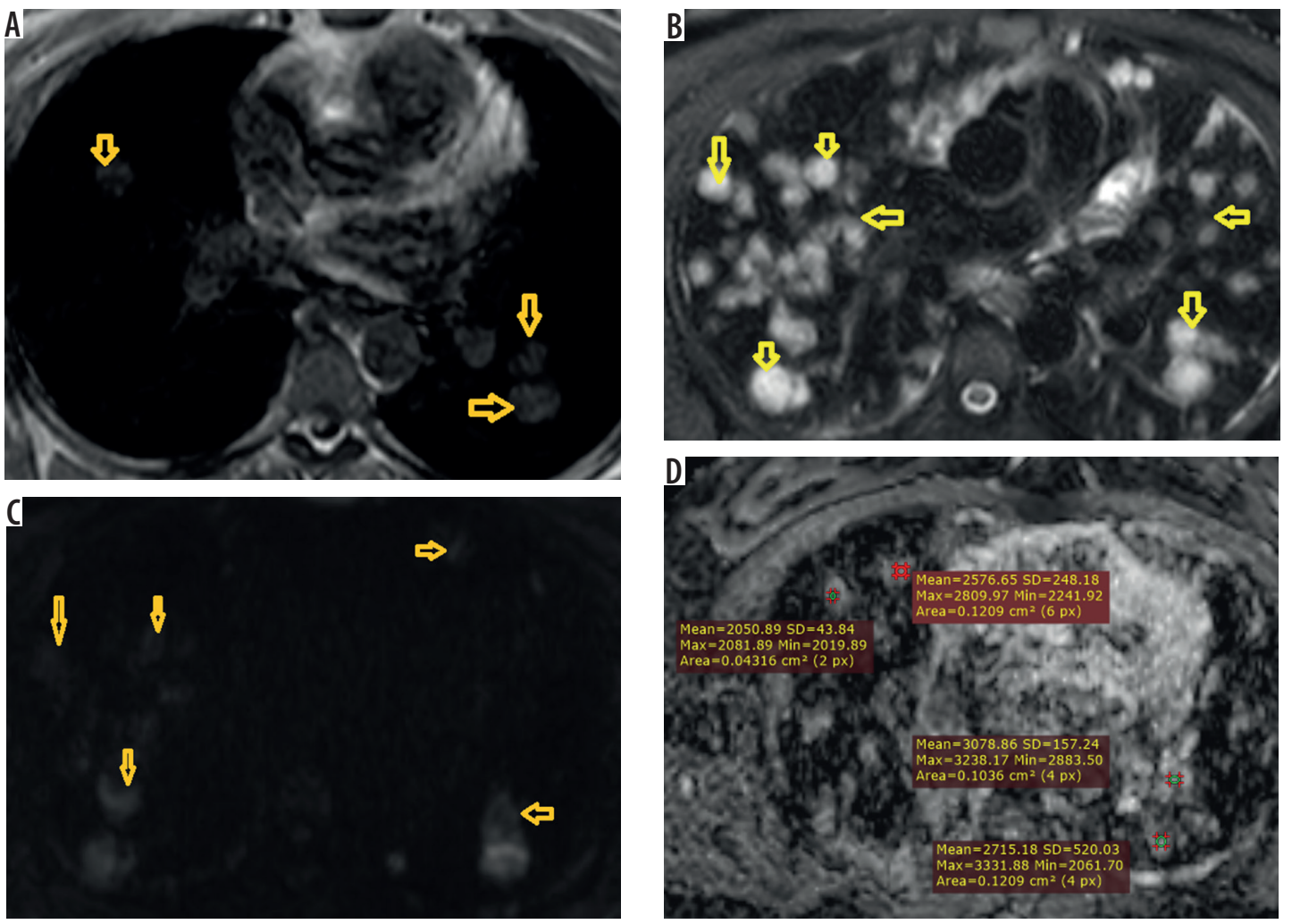

Figure 6.33-year-old female patient presented metastatic adenoid cystic carcinoma of the submandibular gland. A) Axial T1-WI showing multiple bilateral pulmonary nodules with hypointense signal. B) Axial T2-WI with fat saturation showing more delineation of the lesions that show hyperintense signal. C) Axial DWI (b1000) most of the lesions show hypointense signal. D) ADC map showing free diffusion of the lesions with mean $A D C$ value $2.7 \times 10^{-3} \mathrm{~mm}^{2} / \mathrm{s}$. Bright areas on DWI were due to $\mathrm{T} 2$ shine-through artefact
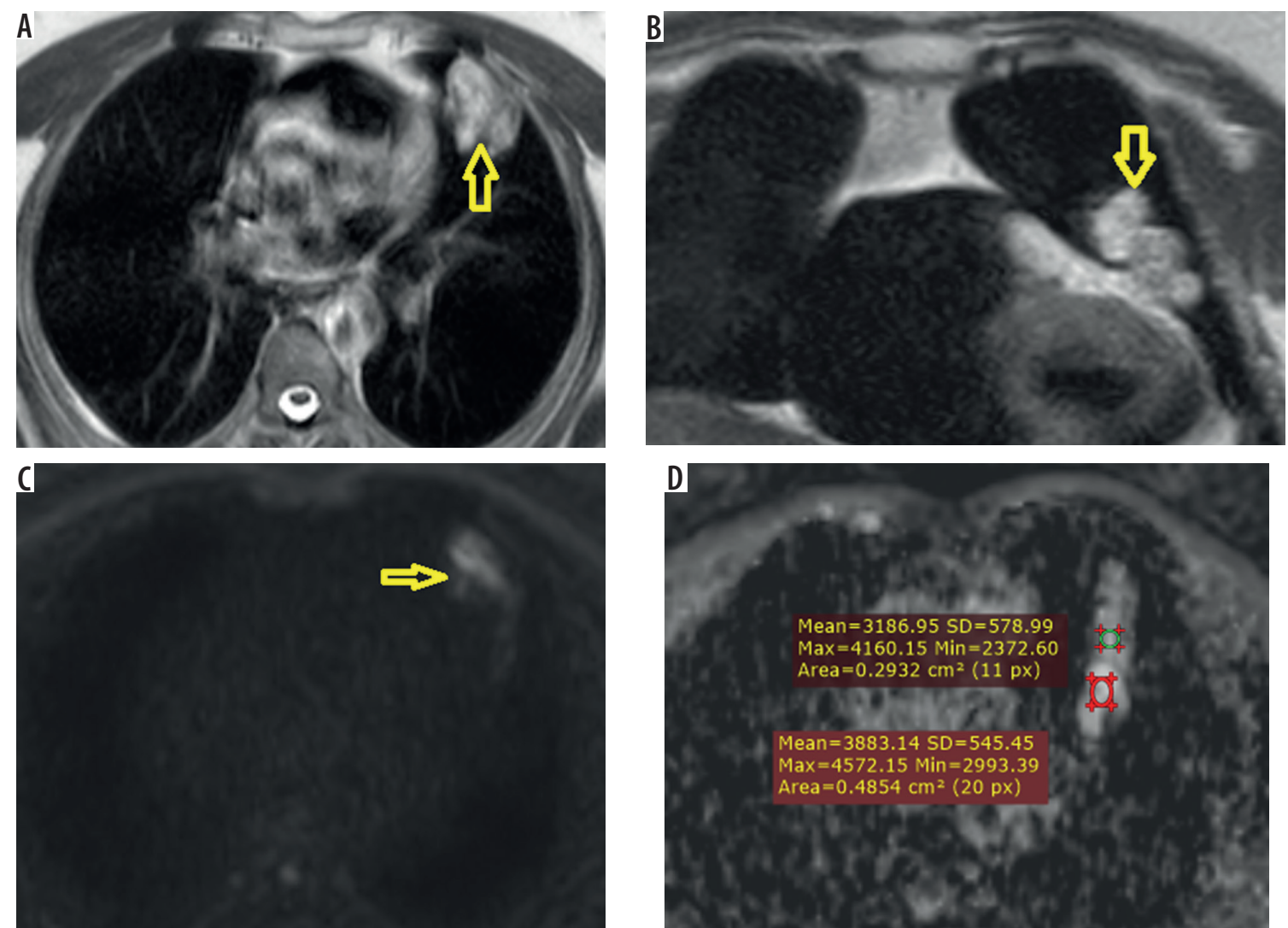

Figure 7. Low-grade neuroendocrine tumour (carcinoid tumour) in 70-year-old female. A) Axial T2-WI showing well-defined mass at the left lung with hyperintense signal. The mass has lobulated smooth outline in Coronal T2-WI (B). C) Axial DWI (b1000) showing hypointense signal of the mass with bright areas. D) $A D C$ map showing free diffusion of the mass with mean $A D C$ value $3 \times 10^{-3} \mathrm{~mm}^{2} / \mathrm{s}$. The bright areas on DWI were due toT2 shine-through effect 

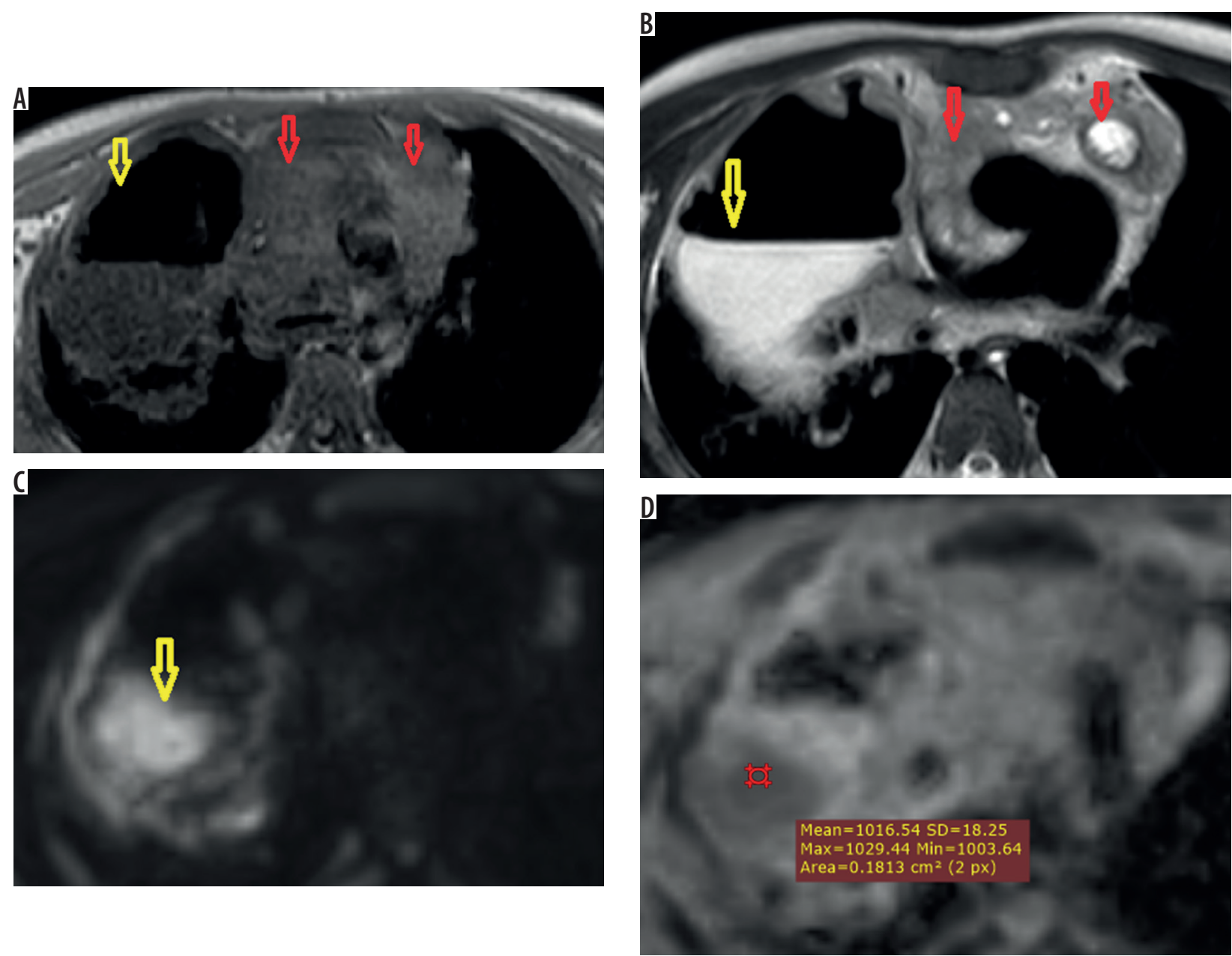

Figure 8. Pyogenic lung abscess in 20-year-old male presented by fever and weight loss. A) Axial T1-WI showing large cavitary lesion at the RT upper lung lobe (yellow arrow) and amalgamated mediastinal LNS (red arrow). B) Axial T2-WI, the cavity shows air fluid level with hyperintense signal of the fluid inside (yellow arrow), and the LNs show central necrosis inside (red arrow). C) Axial DWI (b1000) showing hyperintense signal of the fluid inside the cavity. D) $A D C$ map showing restricted diffusion pattern of contents inside the cavity with mean $A D C$ value $1 \times 10^{-3} \mathrm{~mm}^{2} / \mathrm{s}$

$1.4 \pm 0.51 \times 10^{-3} \mathrm{~mm}^{2} / \mathrm{s}$ and for malignant lesions it was $2.38 \pm 0.3 \times 10^{-3} \mathrm{~mm}^{2} / \mathrm{s}$, which was significantly different from the benign masses; because the benign cystic lesions in our study included pyogenic abscesses (two cases) and a haemorrhagic inflammatory lesion (one case), their contents resulted in diffusion restriction and low ADC value.

Limitations: Our study had several limitations including: difficulty to avoid susceptibility artefacts and artefacts associated with EPI sequences and macroscopic motion on DWI of pulmonary lesions, which can cause image distortion and difficult interpretation of DWI especially in relatively small sized lesions that could be missed due to breathing or subtle motion.

We had a limited number of patients with benign pulmonary lesions, especially soft tissue lesions, because not all benign pulmonary lesions underwent histological confirmation and we included only the pathologically proven cases.

Also, we had a limited number of small cell and squamous cell lung cancer cases, which was not enough for satisfactory and clear results about the difference in the mean ADC value between small cell and non-small cell lung cancer.

There are few data regarding the use of ADC in the classification of different subtypes of bronchogenic carcinoma. Thus, additional high-quality studies are required to evaluate the use of DWI in lung cancer and detect a cut-off value for differentiating different subtypes of malignant pulmonary lesions.

\section{Conclusions}

MR diffusion imaging and measurement of ADC value can help in characterisation of pulmonary lesions, including both solid and cystic lesions. ADC measurement can differentiate between solid benign and malignant lung masses, while measuring the mean ADC value inside the necrotic part of either benign or malignant lesions does not give significant data for their discrimination.

\section{Conflict of interest}

The authors report no conflict of interest. 

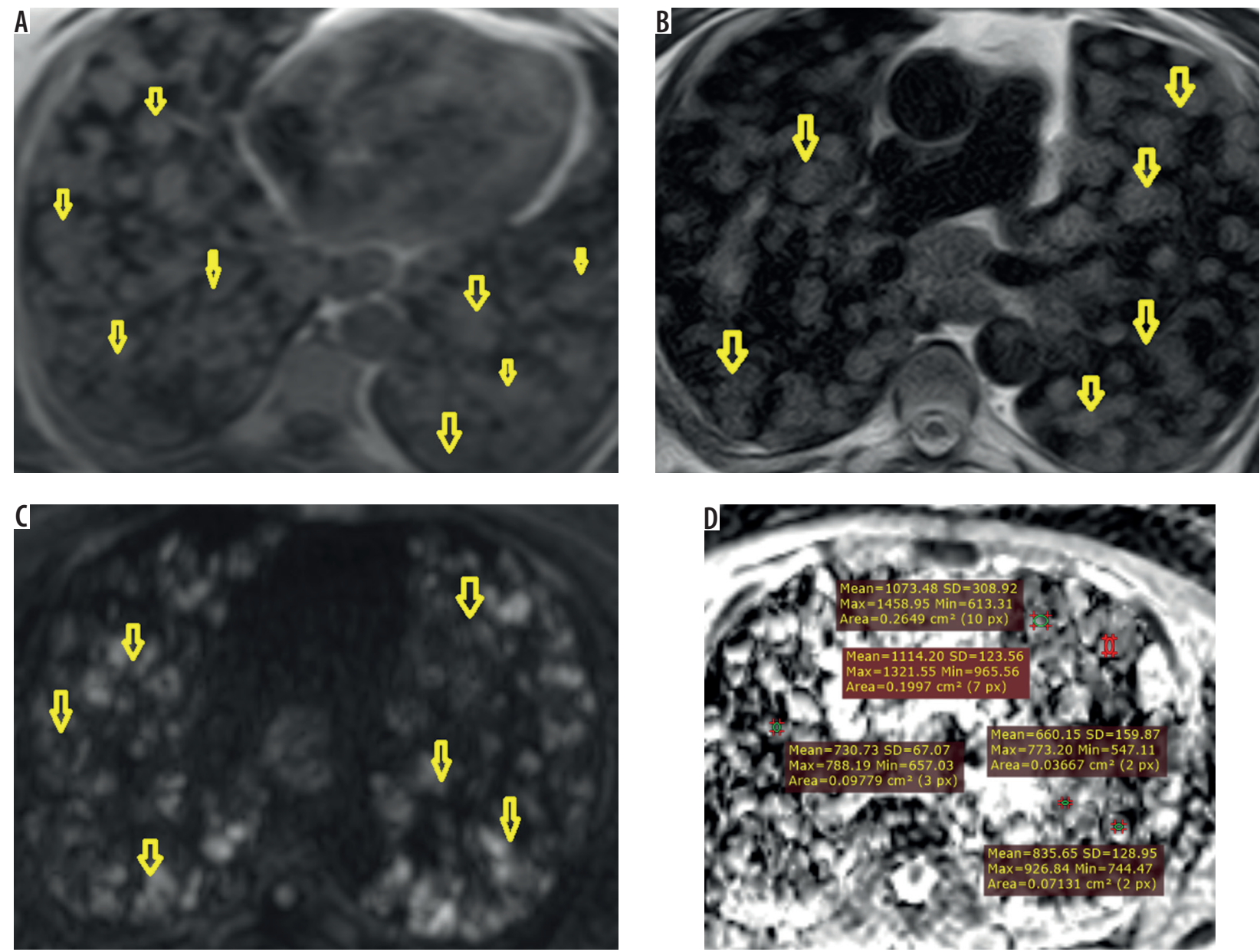

Figure 9. 55-year-old male patient presented metastatic thyroid carcinoma. A) Axial T1-WI showing miliary bilateral pulmonary nodules with hypointense signal. B) Axial T2-WI showing intermediate signal of the lesions. C) Axial DWI (b1000) most of the lesions show hyperintense signal. D) ADC map showing restricted diffusion of the lesions with mean $A D C$ value $0.7 \times 10^{-3} \mathrm{~mm}^{2} / \mathrm{s}$

\section{References}

1. Liu H, Liu Y, Yu T, et al. Usefulness of diffusion-weighted MR imaging in the evaluation of pulmonary lesions. Eur Radiol 2010; 20: 807-815.

2. Satoh S, Kitazume Y, Ohdama S, et al. Can malignant and benign pulmonary nodules be differentiated with diffusion-weighted MRI? AJR Am J Roentgenol 2008; 191: 464-470.

3. Wu L-M, Xu J, Hua J, et al. Can diffusion-weighted imaging be used as a reliable sequence in the detection of malignant pulmonary nodules and masses? Magn Reson Imaging 2013; 31: 235-246.

4. Khalil A, Majlath M, Gounant V, et al. Contribution of magnetic resonance imaging in lung cancer imaging. Diagn Interv Imaging 2016; 97: 991-1002.

5. Qi L, Zhang X, Tang L, et al. Using diffusion-weighted MR imaging for tumor detection in the collapsed lung: a preliminary study. Eur Radiol 2009; 9: 333-341.

6. Bukte Y, Paksoy Y, Genc E, et al. Role of diffusion-weighted MR in differential diagnosis of intracranial cystic lesions. Clin Radiol 2005; 60: 375-383.

7. Whanga J, Kolber M, Powell D, et al. Diffusion-weighted signal patterns of intracranial haemorrhage. Clin Radiol 2015; 70: 909-916.
8. Uto T, Takehara Y, Nakamura Y, et al. Higher sensitivity and specificity for diffusion-weighted imaging of malignant lung lesions without apparent diffusion coefficient quantification. Radiology 2009; 252: 247-254.

9. Sabri Y, Kolta M, Khairy M. MR diffusion imaging in mediastinal masses the differentiation between benign and malignant lesions. Egyptian J Radiol Nucl Med 2017; 48: 569-580.

10. Karaman Y, Ozgür A, Apaydın D. Role of diffusion-weighted magnetic resonance imaging in the differentiation of parotid gland tumors. Japanese Society for Oral and Maxillofacial Radiology and Springer Japan 2015.

11. Abdel Razek A, Elmorsy A, Elshafey M, et al. Assessment of mediastinal tumors with diffusion weighted single shot echo planar MR imaging. J Mag Reson Imaging 2009; 30: 535-540.

12. Gumustas S, Inan N, Sarisoy H, et al. Malignant versus benign mediastinal lesions: quantitative assessment with diffusion weighted MR imaging. Eur Radiol 2011; 21: 2255-2260. 